\title{
Enhanced Performance of a VLC and ULEAPS Fiber Seamless Integrated System Employing Novel Weighted Pre-FDE)
}

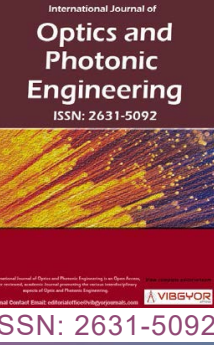

\section{Xiaojun $\mathrm{Yu}^{1}$, Yinaer $\mathrm{Ha}^{1}$, Liangming Xiong ${ }^{2}$, Jie Luo and Nan Chi ${ }^{1^{*}}$}

${ }^{1}$ Key Laboratory for Information Science of Electromagnetic Waves (MoE), Department of Communication Science and Engineering, Fudan University, Shanghai, China

${ }^{2}$ State Key Laboratory of Optical Fibre and Cable Manufacture Technology, Yangtze Optical Fibre and Cable Company Ltd, Wuhan, China

\begin{abstract}
We reported and experimentally demonstrated a $1 \mathrm{Gbit} / \mathrm{s}$ guided visible light transparent based on weighted pre frequency domain equalization (Pre-FDE) and post time domain equalizer over a $100 \mathrm{~m}$ ULEAPS fiber. The experimental results verified the effectiveness and improvement of the proposed method comparing with the traditional post-equalizers. To the best of our knowledge, this is the highest data rate ever achieved in a VLC transparent employing a commercially available blue LED.
\end{abstract}

\section{Index Terms}

Visible light communication, Pre-domain equalizer, Nonlinear distortions

\section{Introduction}

In recent years, there has been growing interest in visible light communication (VLC) due to the rapid development of light emitting diode (LED) technology and the scarcity of spectrum resources [1]. The VLC system offers several advantages such as cost-effective, license-free, electromagnetic interference free and security [2]. As the main technology for next generation illumination and communication, VLC technology has been applied in intelligent home network to make full use of the individual illumination and communication function of optical signals. Increasing request of the communication data rate will drive the peak rates per user up to 1 gigabyte per second (Gbps), thus motivating some new technology in optical communication. Among which the large core $(1 \mathrm{~mm})$ plastic optical fibers (POFs) has attracted great attention for cost reduction and convenient installation. Moreover, LEDs become the optimal choice of source in POFs system since they offer advantages over lasers, such as providing simple coupling and extremely low cost. The integrated system of the POFs and VLC technology can transmit data wirelessly while illuminating rooms. A bit rate of about $8.7 \mathrm{Gbps}$ has been obtained at a BER of for SI-POF lengths of $30 \mathrm{~m}$ by using the SC-MLP equalizer based on RCLED driver [3]. The experiments of M-PAM transmission over $10 \mathrm{~m}$ SI-POF with the MLP based DFE

*Corresponding author: Nan Chi, Key Laboratory for Information Science of Electromagnetic Waves (MoE), Department of Communication Science and Engineering, Fudan University, No. 220, Handan Road, Shanghai, China

Accepted: December 04, 2020; Published: December 06, 2020

Copyright: (c) $2020 \mathrm{Yu} \mathrm{X}$, et al. This is an open-access article distributed under the terms of the Creative Commons Attribution License, which permits unrestricted use, distribution, and reproduction in any medium, provided the original author and source are credited.

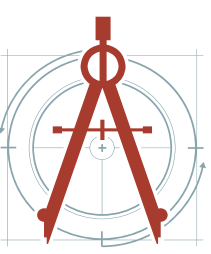

Yu et al. Int J Opt Photonic Eng 2020, 5:027 
(MLP-DFE) was demonstrated with a data rate of $10 \mathrm{Gbit} / \mathrm{s}$ [4]. Furthermore, for better combination with commercial VLC technology, the application of commercial illuminating LEDs on guided VLC transmission was experimentally demonstrated later in 2018 [5]. To meet the long range and multi-function communication, the combined applications of commercial illuminating LEDs and ULEAPS fiber are the attracting research filed nowadays.

On the other hand, for mitigating the severe frequency response of VLC system, discrete multitones (DMT) and orthogonal frequency division multiplexing (OFDM) have already been widely employed in VLC signal modulation [6]. In [2], pre and post frequency equalizers were used to compensate the LED distortion in a bi-directional OFDM experiment. In [7], optimized DMT modulation was employed to a indoor WDM VLC transmission to achieve the high data rate of $3.4 \mathrm{Gbit} / \mathrm{s}$. The OFDM signal can be easily equalized in frequency domain, however, it suffers from high peak-to-average power ratio (PAPR), frequency offset, and phase-noise sensitivities [8]. An alternative promising approach to distortion mitigation is the use of SC-FDE, which achieves comparable performance of OFDM, while avoiding the disadvantages associated with multicarrier (MC).

In this paper, for the first time, we employed a combination of weighted pre-frequency domain equalizer and post time domain equalizer based on least mean square and Volterra series (LMS + Volterra) to resist severe nonlinear distortions. Zero-forcing pre-equalization in frequency domain can compensate the received signal spectrum more likely to a flat transmitted signal, however, considering the unique channel feature in VLC system such as constrained peak and total power, it's not the most suitable and reasonable pre-equalization method. Thus, we implemented a weighted pre-equalizer to meet the requirement of equalization and channel specialty simultaneously in a guided 16 carrier less amplitude and phase (16-CAP) seamless VLC transparent over a $100 \mathrm{~m}$ Ultra-Large Effective Area (ULEAPS) fiber. Experimental results illustrate that the proposed equalizer can obtain a higher measured $\mathrm{Q}$ factor by $1.5 \mathrm{~dB}$ than that of post linear and nonlinear equalizers. Moreover, $1 \mathrm{Gbit} / \mathrm{s}$ data rate can be achieved over $100 \mathrm{~m}$ ULEAPS fiber with the target bit error rate (BER) of (assumed as forward error correction (FEC) limit [9]) by using the proposed combined equalization while only 0.93 Gbit/s data rate by traditional post LMS + Volterra equalizer. To the best of our knowledge, this is the highest reachable data rate in a guided VLC transparent system with a single commercial illumination LED.

\section{Principle}

The block diagram and overall test setup of the
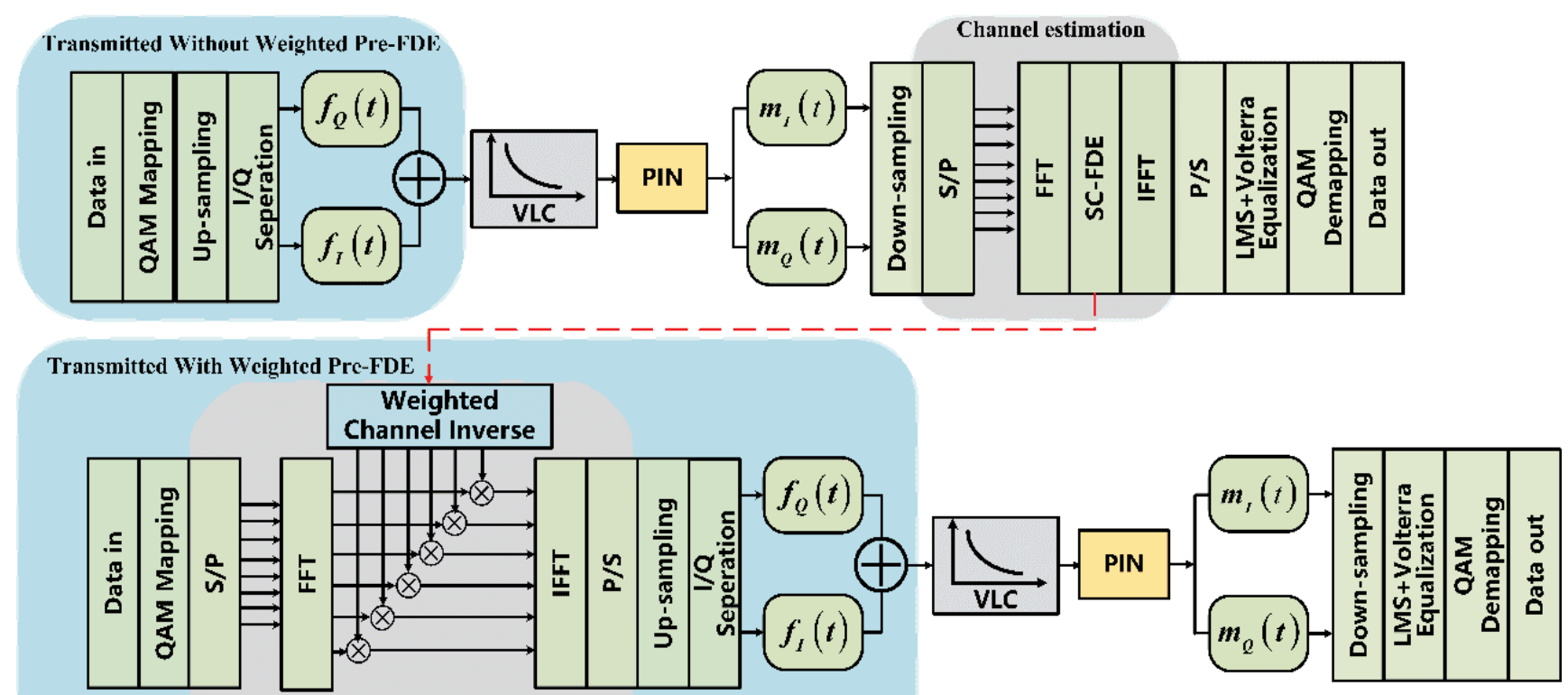

Weighted Pre-FDE

Figure 1: Schematic diagram of the weighted pre-FDE and post timing LMS + Volterra equalizer based on 16-CAP VLC system. 
proposed weighted pre-FDE and post timing equalizer based on 16-CAP VLC system is demonstrated as Figure 1. The whole proposed scheme can be divided into two stage: Training mode and detecting mode. Firstly in training mode, signal was transmitted without pre-FDE, the distorted frequency response of received signal was compensated by FDE via zero forcing (ZF) algorithms at the receiving end to obtain and update the channel estimation simply for pre-FDE as it shown in Equ. (1).

$$
H_{Z F}=\frac{T s}{R s}
$$

Here, Ts was the original transmitted frequency signal in block FFT size. Relatively, Rs was the received frequency signal without pre-FDE in block FFT size. $H_{Z F}$ was the channel estimation. After that, in detecting mode, $H_{Z F}$ was sent to the preFDE module for equalize the mapping signal. To avoid the phase offset and meet the constrained peak and total power, we use the weighted absolute value of $H_{Z F}$ as the pre-factor rather than the origin value of $H_{Z F}$. The choice of pre-factor weight $\alpha$ can be obtained from the real channel condition. In our paper, $\alpha$ is set to the square root of channel inverse.

For more clearly and intuitionistic explanation of the proposed system, the frequency spectra of transmitted and received signal under different condition with 0.205 roll-off factor in a data rate of
$0.83 \mathrm{Gbit} / \mathrm{s}$ were illustrated in Figure 2 Compared with the origin transmitted signal (Figure 2a), the transmitted signal after weighted pre-FDE (Figure $2 b)$ and ZF pre-FDE signal (Figure 2e) attenuate low frequency parts and amplify high frequency parts to compensate the whole receiving spectrum more likely to the transmitted signal to get a better system performance, which can be seen in the received signal spectra in different transmitted situation in Figure 2. The origin received signal elucidates the large difference between low and high frequency parts due to the channel response of VLC system in Figure 2c. Pre-FDE transmitted signal can compensate the channel distortion, ZF pre-FDE can lead to the most flat received spectrum as it shown in Figure $2 \mathrm{f}$ among the three transmitted method, nevertheless, the PIN receivers cannot detect low frequency components with higher signal to noise ratio (SNR) linearly [10] which causes worse receiving performance.

Consequently, we proposed weighted pre-FDE to partial pre-equalization. The received frequency response of weighted pre-FDE transmitted signal is shown in Figure $2 d$, which obtains relatively small deviation between low and high parts and mitigate the channel distortion.

\section{Experimental Configuration}

Figure 3 illustrates the block diagram of the proposed transmission system setup used in the fol-

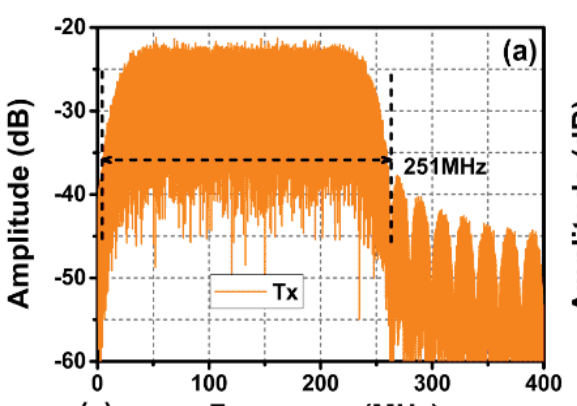

(a) Frequency $(\mathrm{MHz})$

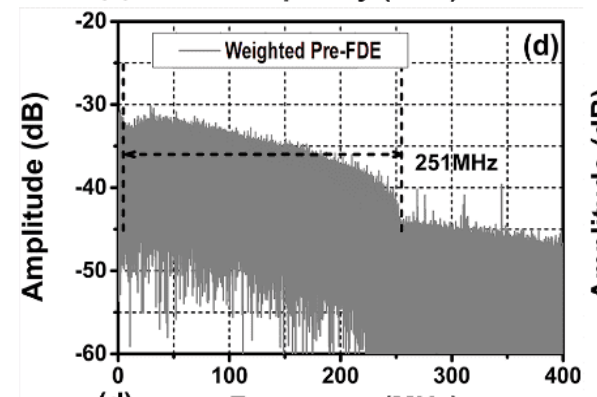

(d)

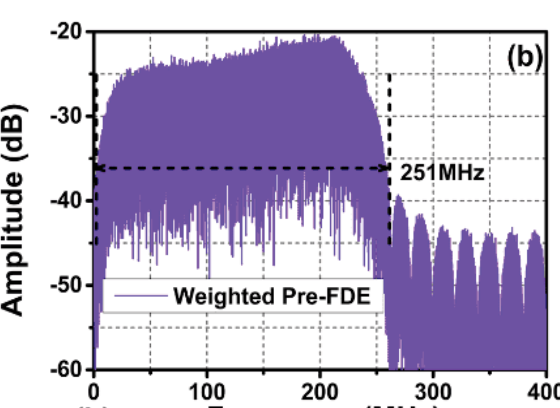

(b)

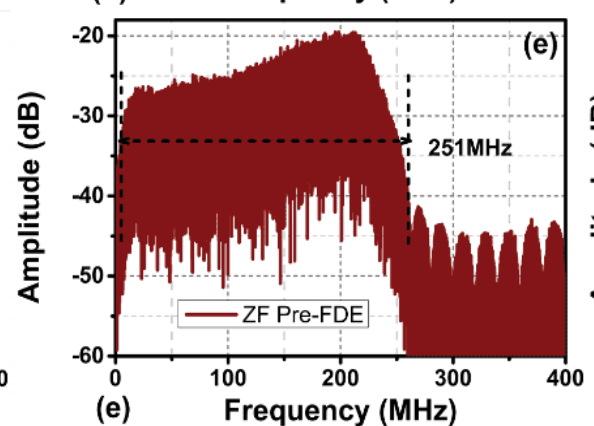

(e) Frequency $(\mathrm{MHz})$
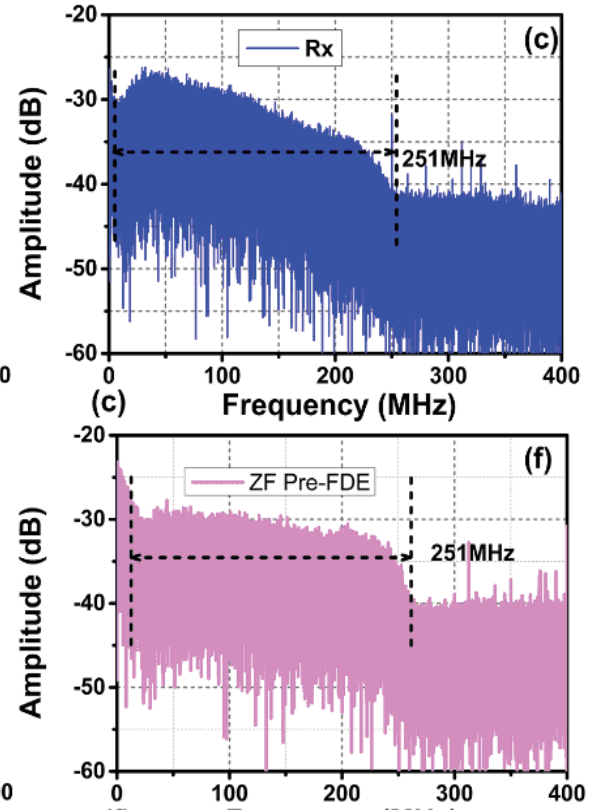

(f) Frequency $(\mathrm{MHz})$

Figure 2: The transmitted signal and received signal frequency spectra employing three equalization methods. 


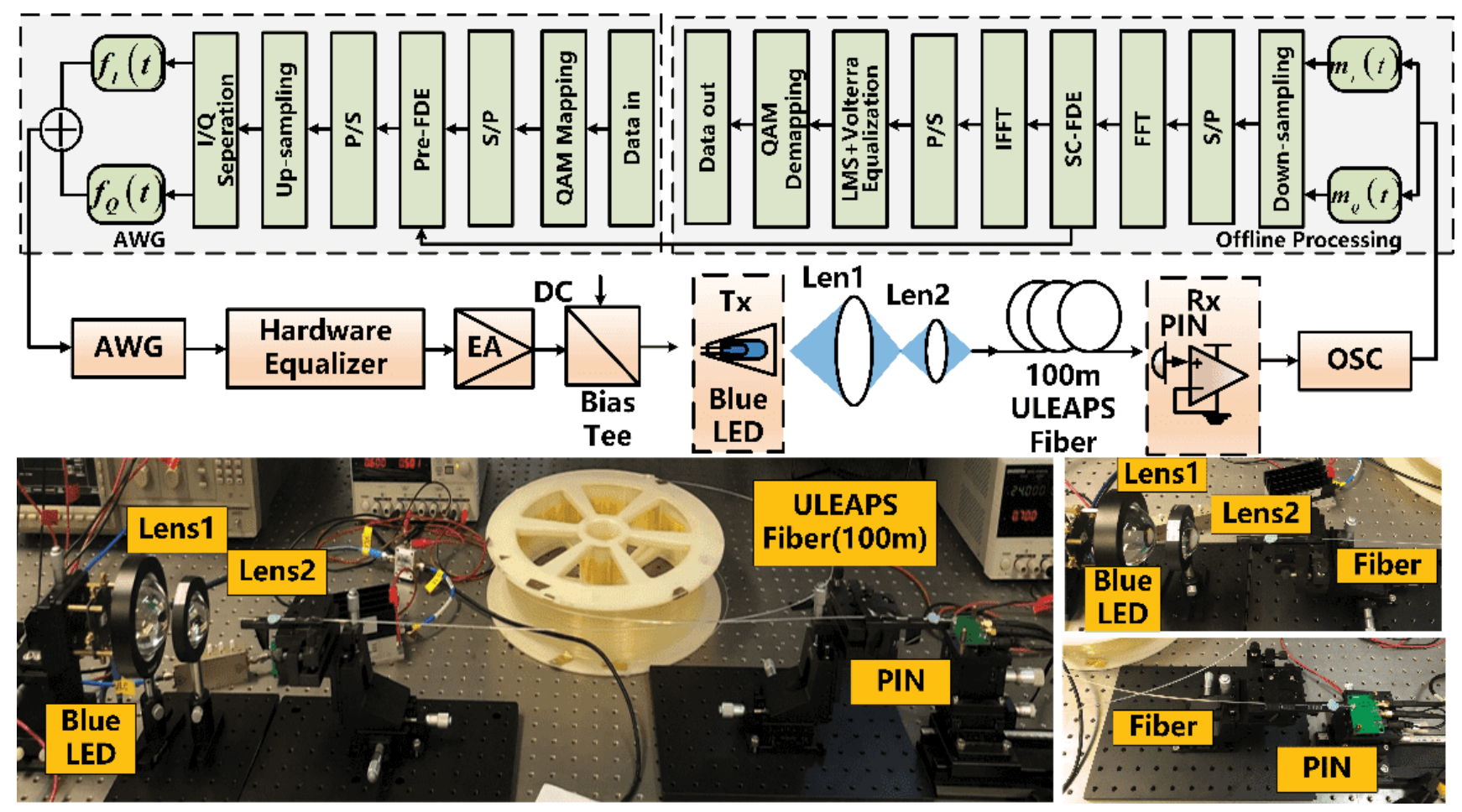

Figure 3: Experimental setup and block diagram of guided VLC system over ULEAPS fiber.
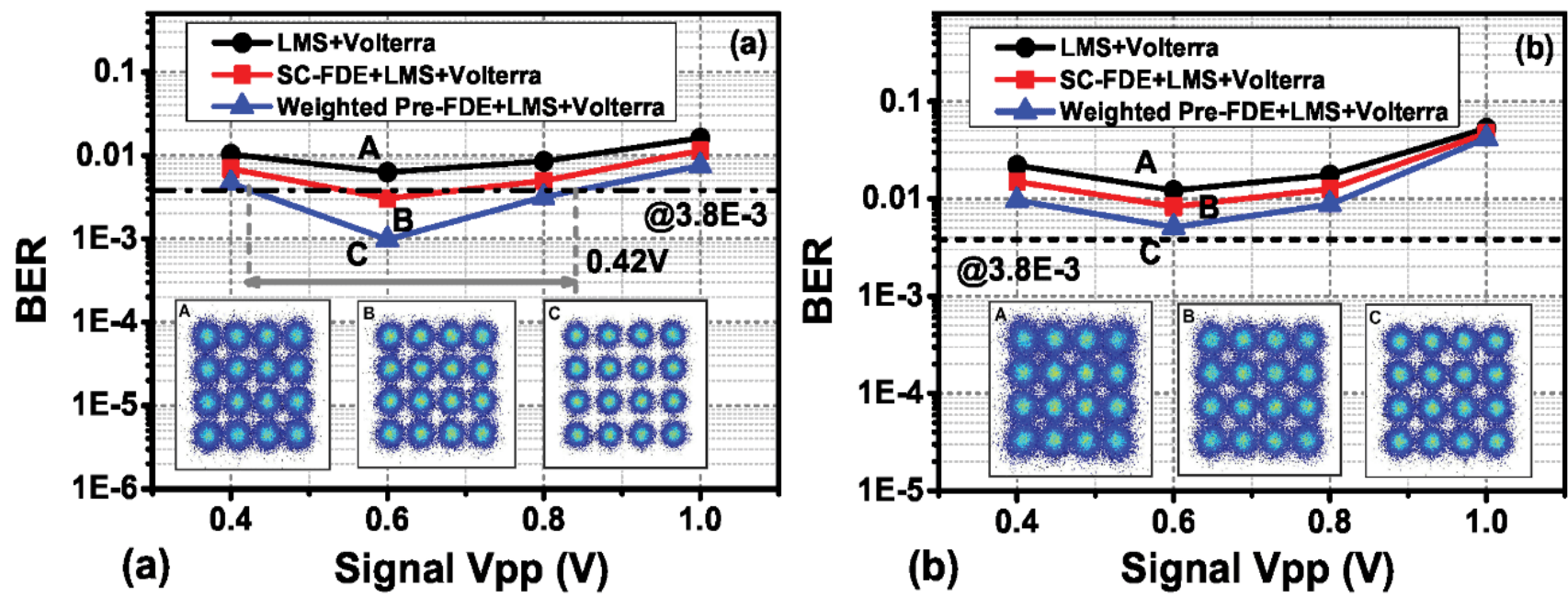

Figure 4: Measured BER results versus input signal Vpp with different equalizer with $160 \mathrm{~mA}$ bias current a) At date rate of $0.93 \mathrm{Gbit} / \mathrm{s}$; b) At date rate of $1 \mathrm{Gbit} / \mathrm{s}$.

lowing experiments. At the transmitting end, the binary information data was firstly mapped and coded by 16-ary quadrature amplitude modulator (16-QAM). After mapping, transmitted data was converted from series to parallel to prepare for the block of pre-frequency equalization module. After making pre-equalization, the signal was up-sampled by a factor of 6 and filtered by a rectangular filter. Signal was then applied to an Arbitrary Waveform Generator (AWG710) and was filtered by a bridged-T based hardware filter. After that, an electrical amplifier (EA) and a Bias Tee was used to amplify the optical signal. Subsequently, the transmitting signals were delivered by a commercial blue LED (RGB LED, Engine LZ4-20MA00). With the coupling function of two lens, the optical signals were imported in a Yangtze Optical Fiber and Cable Company Ltd produced $100 \mathrm{~m}$ ULEAPS fiber.

At the ULEAPS fiber end, the 16-CAP signal was directed detected by a commercial positive intrin- 
sic-negative (PIN). To reduce the complexity and improve the exactitude of the experiment, PIN receiver need to locate closely to the optical fiber end without lens coupling. A digital storage oscilloscope with $2 \mathrm{Gs} / \mathrm{s}$ sampling rate recorded the outputs of the PIN receiver for further offline processing.

\section{Results and Discussions}

To acquire the best working point, we initially make the traversal of LED current and input signal Vpp. Figure 4 and Figure 5 respectively demonstrate the measured BER versus signal $\mathrm{Vpp}$ under different equalization methods under $160 \mathrm{~mA}$ and $200 \mathrm{~mA}$ bias LED current. In Figure 4, post LMS linear and Volterra nonlinear equalizer cannot decode the signal correctly under a low LED current of 160 $\mathrm{mA}$. As for the two-stage post frequency and time domain equalizer, it accomplishes the $7 \%$ forward error correction (FEC) limit only at the best operate point $0.6 \mathrm{~V}$. However, the proposed weighted preFDE and post LMS + Volterra equalizers achieve a substantial decrease of BER which allow the system work between $0.42 \mathrm{~V}$ and $0.84 \mathrm{~V}$ signal Vpp under the error threshold at the data rate of $0.93 \mathrm{Gbit} / \mathrm{s}$ in Figure 4a. When the LED current increasing to $200 \mathrm{~mA}$, As we can see, these three kinds of equalization can reach the threshold of BER below 7\% forward error correction (FEC) limit at $0.93 \mathrm{Gbit} / \mathrm{s}$ data rate, among which the proposed pre and post equalizers perform better than others with $0.5 \mathrm{~V}$ working range in Figure $5 a$. In Figure 5b, only the proposed weighted pre-FDE and post time domain equalizer achieve BER below the error threshold at $1 \mathrm{~Gb} / \mathrm{s}$ under signal Vpp $=0.6 \mathrm{~V}$. The constellations of the optimal working signal Vpp employing three methods of equalization are also carried out in Figure 4 and Figure 5, among which the proposed has the clearest constellation.

Figure 6 illustrates a traversal of data rate under four representative signal $\mathrm{Vpp}$ under optimal LED working current $200 \mathrm{~mA}$. At a relatively low signal power point $0.4 \mathrm{~V}$, the proposed scheme outperform the other two post equalizers by increasing about $65 \mathrm{Mbit} / \mathrm{s}$ data rate in Figure $6 \mathrm{a}$. In the best signal Vpp condition $0.6 \mathrm{~V}$, the weighted pre-FDE and post $\mathrm{LMS}+$ Volterra equalizer can lead to a highest data rate of $1 \mathrm{Gbit} / \mathrm{s}$ while only $0.93 \mathrm{Gbit} / \mathrm{s}$ for LMS + Vloterra equalizer and $0.97 \mathrm{Gbit} / \mathrm{s}$ for SC-FDE and LMS + Vloterra equalizer in Figure 6b. However, in high nonlinear distortion point $0.8 \mathrm{~V}$ and $1.0 \mathrm{~V}$, the reachable data rate decrease to 0.97 Gbit/s in Figure $6 \mathrm{c}$ and $0.9 \mathrm{Gbit} / \mathrm{s}$ in Figure $6 \mathrm{~d}$ relatively through the proposed method. Despite that, the proposed also has the highest data rate among three kinds of equalization.

To numerical measure the enhance performance of our proposed method, the $Q$ factor was calculated versus different data rate and signal Vpp with three different equalization methods under a fixed LED optimal current $=200 \mathrm{~mA}$ in Figure 7. It is easily to conclude that the proposed weighted preFDE and post LMS + Volterra equalization methods can improve the $Q$ factor of the LMS + Volterra filter by $1.5 \mathrm{~dB}$ and outperform the SC-FDE and LMS + Volterra filter by $0.9 \mathrm{~dB}$ at $0.83 \mathrm{Gbit} / \mathrm{s}$ in Figure 7a. As for the optimal working signal Vpp, the performance of our proposed equalizer is improved by

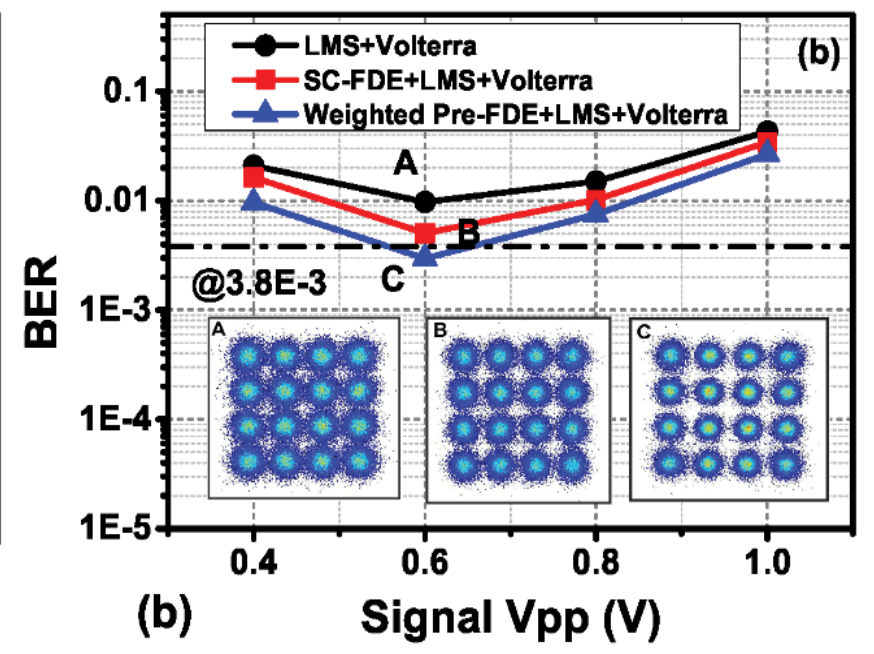

Figure 5: Measured BER results versus input signal Vpp with different equalizer with $200 \mathrm{~mA}$ bias current a) At date rate of $0.93 \mathrm{Gbit} / \mathrm{s}$; b) At date rate of $1 \mathrm{Gbit} / \mathrm{s}$. 

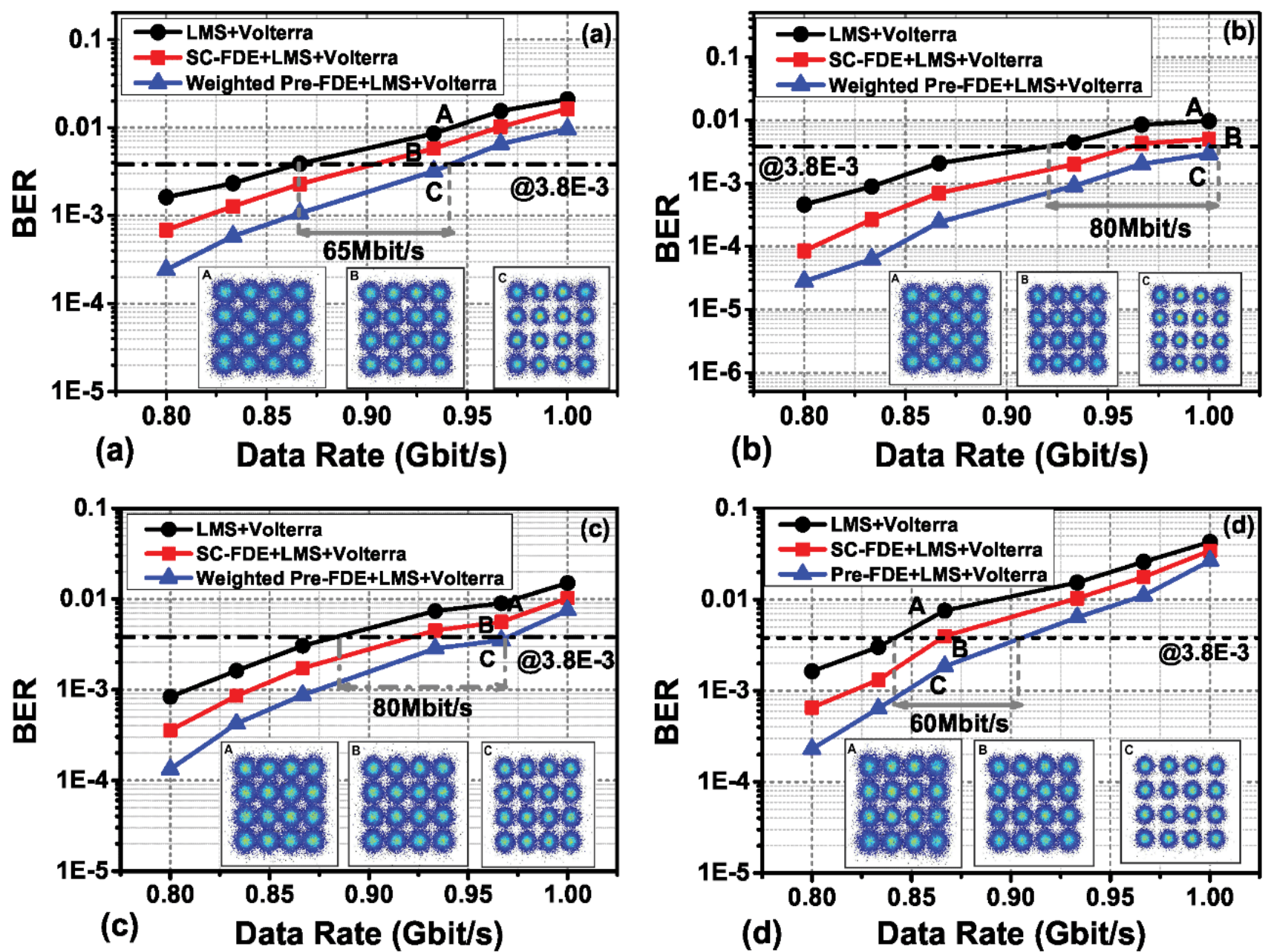

Figure 6: Measured BER results versus Data Rate employing different equalizer for Blue LED over 100m fiber a) At $0.4 \mathrm{~V}$; b) At $0.6 \mathrm{~V}$; c) At $0.8 \mathrm{~V}$; d) At $1.0 \mathrm{~V}$.
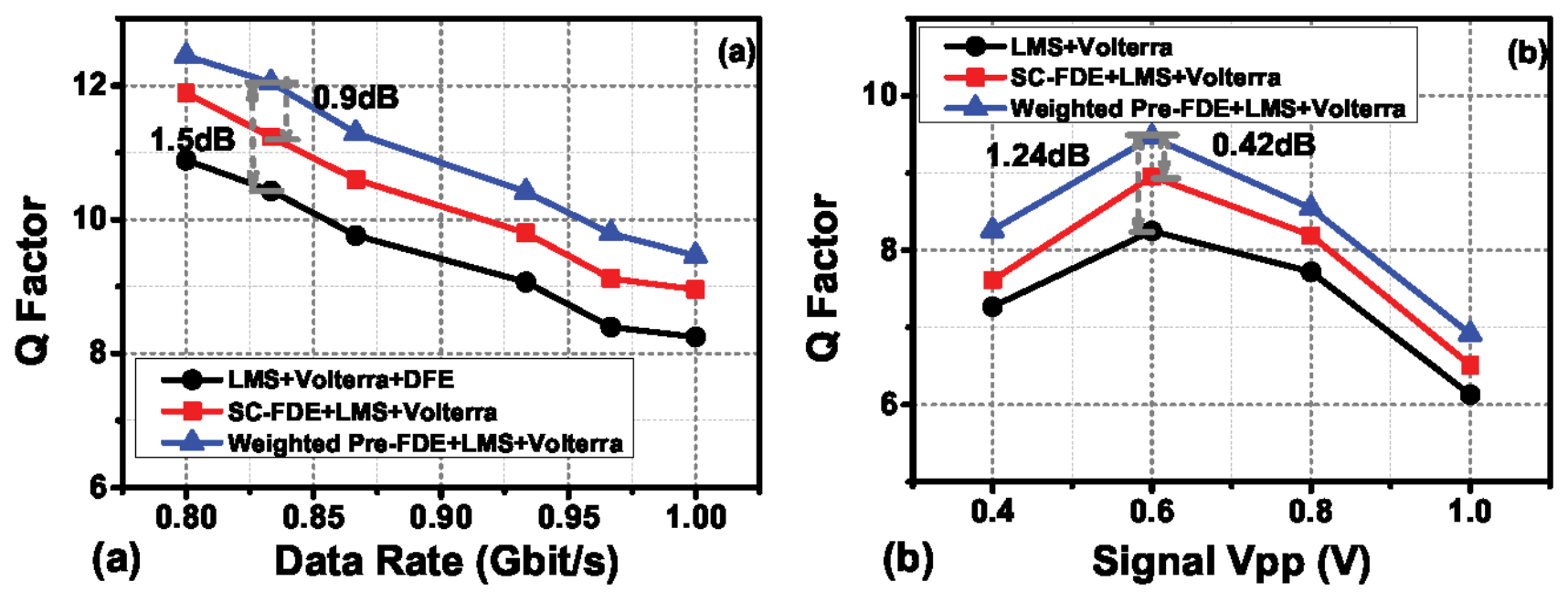

Figure 7: Q-factor comparison between different equalization methods: a) With different Data Rate; b) With different signal Vpp. 
1.24 dB over the LMS linear + Volterra nonlinear equalizer and by $0.42 \mathrm{~dB}$ over the SC-FDE and LMS + Volterra equalizer.

\section{Conclusions}

In this paper, to remove the nonlinear distortion caused by Inter Symbol Interference (ISI), we experimentally demonstrated a hybrid weighted pre frequency domain and post time domain equalization. The enhance performance of the proposed scheme can be obtained through an experimental demonstration based on 100m ULEAPS fiber seamless VLC integrated transparent using a commercial blue LED. As the results show, the proposed preFDE and post equalization can perform better than the traditional post time domain equalizer by $Q$ factor of $1.5 \mathrm{~dB}$ and successfully increase the data rate from $0.93 \mathrm{Gbit} / \mathrm{s}$ to $1 \mathrm{Gbit} / \mathrm{s}$. As far as we know, this is the highest data rate when investigated the VLC transparent from free-space to guided ULEAPS fiber. By using this scheme, nonlinear dispersion can be reduced effectively and the resolution of LOS transmission drawbacks for VLC system.

\section{Acknowledgment}

This work was partially supported by the $\mathrm{Na}$ tional Key Research and Development Program of China (2017YFB0403603), the NSFC project (No.61925104).

\section{References}

1. N Chi, H Haas, M Kavehrad, TDC Little, XL Huang (2015) Visible light communications: Demand factors, benefits and opportunities [guest editorial]. IEEE Wireless Commun 22: 5-7.

2. Yuanquan Wang, Yiguang Wang, Nan Chi, Jianjun Yu, Huiliang Shang (2013) Demonstration of $575-\mathrm{mb} / \mathrm{s}$ downlink and 225-mb/s uplink bi-directional SCM-
WDM visible light communication using RGB LED and phosphor-based LED. Opt Express 21: 1203-1208.

3. IN Osahon, S Rajbhandari, WO Popoola (2018) SI-POF transmission with CAP modulation and split-complex MLP equalizer. 2018 IEEE International Conference on Communications (ICC), Kansas City, MO, USA.

4. IN Osahon, M Safari, WO Popoola (2018) 10-Gb/s transmission over 10-m SI-POF with $\mathrm{M}$-pam and multilayer perceptron equalizer. IEEE Photonics Technology Letters 30: 911-914.

5. J Shi, Y Zhou, N Chi, L Xiong, J Luo (2018) Gigabit LEDbased visible light transparent transmission from free-space to a 100-m ultralarge effective area pure silica fiber. Microwave and Optical Technology Letters 60: 13-18.

6. Y Wang, J Shi, C Yang, Y Wang, N Chi (2014) Integrated $10 \mathrm{~Gb} / \mathrm{s}$ multilevel multiband passive optical network and $500 \mathrm{Mb} / \mathrm{s}$ indoor visible light communication system based on Nyquist single carrier frequency domain equalization modulation. Opt Lett 39: 2576-2579.

7. G Cossu, AM Khalid, P Choudhury, R Corsini, E Ciaramella (2012) 3.4 Gbit/s visible optical wireless transmission based on RGB LED. Opt Express 20: B501-B506.

8. N Chi, Y Wang, Y Wang, X Huang, X Lu (2014) Ultrahigh-speed single red-green-blue light-emitting diode-based visible light communication system utilizing advanced modulation formats. Chinese Optics Letter 12: 22-25.

9. CF Reed (1999) Error-Control Coding for Data Networks. Springer, US.

10.KJ Williams, LT Nichols (1998) Photodetector nonlinearity limitations on a high-dynamic range $3 \mathrm{GHz}$ fiber optic link. Journal of Lightwave Technology 16: 192-199. 